\title{
METODOLOGIA PBL COMO ALTERNATIVA PARA VIABILIDADE DO ENSINO NO CONTEXTO DE PANDEMIA DA COVID-19
}

Luamim Sales Tapajós - luamim.tapajos@ufopa.edu.br*

Universidade Federal do Oeste do Pará, Campus de Itaituba, Curso de Engenharia Civil* Rua Universitária, S/N, Bairro Maria Madalena*

68183-300 - Itaituba - Pará*

Valquíria Santana da Silva - valquiriasantana2002@gmail.com*

Universidade Federal do Oeste do Pará, Campus de Itaituba, Curso de Engenharia Civil*

Rua Universitária, S/N, Bairro Maria Madalena*

68183-300 - Itaituba - Pará*

Fabiano Hector Lira Muller-fabiano.muller@ufopa.edu.br

Universidade Federal do Oeste do Pará, Campus de Itaituba, Curso de Engenharia Civil* Rua Universitária, S/N, Bairro Maria Madalena *

68183-300 - Itaituba - Pará*

Ronne Clayton de Castro Gonçalves - ronne.goncalves@ufopa.edu.br

Universidade Federal do Oeste do Pará, Campus de Itaituba, Curso de Engenharia Civil* Rua Universitária, S/N, Bairro Maria Madalena *

68183-300 - Itaituba - Pará*

Andryo Henrique Freitas da Silva - andryoengcivil@ hotmail.com

Faculdade de Itaituba, Curso de Engenharia Civil

Avenida Governador Fernando Guilhon, 895, Bairro Jardim das Araras

68180-110 - Itaituba - Pará*

Resumo: Este trabalho apresenta uma revisão bibliográfica sobre a utilização da metodologia PBL em cursos de Engenharia Civil, assim como traz um contexto do próprio curso de Engenharia Civil no município de Itaituba-PA, para possibilitar melhor compreensão nos desafios envolvidos para adoção desta ferramenta nessa realidade. O objetivo principal deste trabalho se concentra na adoção do PBL como alternativa para continuidade do ensino no contexto da pandemia pela Covid-19, assim como a possibilidade e os desafios para utilização desse método dentro dos cursos de Engenharia Civil ofertados no município de Itaituba. Para obtenção dos resultados, foram realizadas ações envolvendo duas IES, por meio da produção de totens para álcool em gel, de modo que possibilitasse o desenvolvimento dos trabalhos pelos alunos das duas instituições, gerando um produto para a sociedade. Concluiu-se que a metodologia PBL é uma ferramenta eficiente para possibilitar um ensino de qualidade no contexto de pandemia e pós-pandemia e que, apesar dos desafios, é possível utilizá-la nos dentro dos cursos estudados.

Palavras-chave: Metodologia PBL. Engenharia Civil. Pandemia. Itaituba. 


\section{INTRODUÇÃO}

Atualmente a globalização está cada vez mais comum e frequente, afetando todos os âmbitos da sociedade, entre eles está o mercado de trabalho, que se encontra bem mais exigente quanto à contratação de novos profissionais, por essa razão novos métodos de ensino e/ou aprendizagem estão sendo criados e aplicados, inclusive nos cursos de Engenharia, com o intuito de suprimento dessa exigência. No entanto, segundo Masson et al. (2012), o professor universitário está encontrando dificuldades para criar e implementar novas tecnologias de ensino, que desenvolvam esses saberes e fazeres e ao mesmo tempo reduzam o tempo de permanência do aluno em sala de aula e aumentem a sua eficiência, porém o mesmo afirma que o PBL é uma abordagem sistêmica, que envolve os alunos na aquisição de conhecimentos e competências por meio de um processo de investigação de questões complexas, tarefas autênticas e produtos cuidadosamente planejados com vista a uma aprendizagem eficiente e eficaz.

De acordo com Ribeiro (2005), a Engenharia exerce papel fundamental no funcionamento e desenvolvimento da sociedade, e tem sido uma das áreas mais afetadas pelas céleres transformações tecnológicas, que por sua vez tem gerado novas expectativas em relação à prática do engenheiro, bem como no ensino de Engenharia dos cursos superiores, para que a atuação deste profissional não se torne obsoleta. Segundo pesquisas de Macambira (2011) foi possível constatar o que a $\mathrm{ABP}$ (ou PBL) favorece o desenvolvimento de atributos essenciais para a vida profissional dos futuros engenheiros, tais como a adaptabilidade a mudanças, habilidade de solucionar problemas em situações não rotineiras, pensamento crítico e criativo, adoção de uma abordagem sistêmica, trabalho em equipe, capacidade de identificação de pontos fortes e fracos e compromisso com o aprendizado e aperfeiçoamento contínuos.

Dentro desse contexto, o cenário durante a pandemia causada pela Covid-19 aumenta a preocupação quanto à forma de retorno presencial às atividades acadêmicas e estratégias para viabilizar o ensino remoto, visto que, por medidas de segurança, é recomendado que haja a redução do número de pessoas em espaços fechados, como as salas de aula. Dessa forma, metodologias ativas de ensino, como o PBL, podem contribuir para a redução do tempo em que os alunos passam dentro das salas de aula, bem como reduzem os grupos de estudos para desenvolver determinadas atividades, evitando aglomerações.

Além disso, outra vantagem da metodologia PBL é quanto à capacitação do aluno para desenvolver soluções para diversos tipos de problemas de forma autônoma e em conjunto, sendo que, para Ribeiro (2005), habilidades de trabalho em equipe e comunicação são fundamentais para os engenheiros. No entanto, o mesmo autor cita como dificuldade para adoção da metodologia o tempo para execução das atividades consumido pelos alunos. Macambira (2011) também menciona que uma das dificuldades da implantação do PBL nas atividades de ensino ocorre pela necessidade de que o professor certo conhecimento político, econômico e social da cidade e região em que a turma se encontra.

Quando se trata das dificuldades na implantação da metodologia PBL como ferramenta de ensino, é possível citar particularidades encontradas na Amazônia, principalmente nas cidades do interior. Vieira (2017) aponta que poucos estudos sobre a adoção do PBL são produzidos na Região Norte. Ressalta-se, também, que na maioria das cidades do interior da Amazônia boa parte dos professores das IFES são originários de outras cidades, dificultando a inserção em alguns pontos daquela região, como menciona Macambira (2011). Além disso, geralmente, nessas cidades, o acesso à internet é de baixa qualidade, dificultando a realização de atividades remotas. 
Com base nisso, este artigo tem como objetivo avaliar os pontos positivos e negativos da implantação da metodologia PBL nos componentes curriculares dos cursos de Engenharia, contribuindo para o estudo de viabilidade da adoção dessa metodologia nos cursos de Engenharia Civil da Universidade Federal do Oeste do Pará, ofertado no Campus de Itaituba, cidade localizada na região sudoeste paraense, e também da Faculdade de Itaituba (FAI).

\section{REVISÃO BIBLIOGRÁFICA}

\subsection{Metodologia PBL}

A metodologia PBL (Problem Based Lerarning) ou ABP (Aprendizagem Baseada em Problemas/Projetos), traduzindo para o português, foi reconhecida, segundo estudos, como uma metodologia de ensino na Universidade McMaster no Canadá, por volta de 1970, porém apenas nos cursos de Medicina (VIEIRA, 2017). No entanto, essa metodologia vem ganhando espaço em muitas Universidades de diversos países, inclusive no Brasil.

Essa metodologia apresenta resultados benéficos com relação à construção de futuros profissionais, entre estes estão os engenheiros, que lidam constantemente com resolução de problemas. O PBL atua de forma inovadora, mesclando a aprendizagem teórica com a prática para melhor visualização dos problemas que surgem durante a execução das atividades, sejam essas na vida acadêmica ou no mercado de trabalho.

O ciclo de trabalho com um problema no PBL ocorre da seguinte maneira: a) introdução e definição do problema; b) levantamento de hipóteses; c) tentativa de solução com os conhecimentos disponíveis; d) levantamento de pontos de aprendizagem; e) planejamento do trabalho em grupo; f) estudo independente; g) compartilhamento de informações no grupo; h) aplicação dos conhecimentos no problema; i) apresentação das soluções do grupo; j) auto avaliação, avaliação do processo e de pares (Ribeiro, 2008). Nessa metodologia não importa apenas os acertos ou erros encontrados e cometidos pelos discentes durante a apresentação da resolução dos problemas, mas a quantidade e qualidade das pesquisas realizadas.

\subsection{Adoção da Metodologia PBL nos cursos de Engenharia Civil}

Neves e Formoso (2007) desenvolveram um estudo para responder ao questionamento: "Como desenvolver as competências dos gerentes intermediários na indústria da construção civil, utilizando-se a Aprendizagem Baseada em Problemas (ABP)?”. O estudo envolveu alunos de graduação em Engenharia Civil da Universidade Federal do Rio Grande do Sul e da Universidade do Vale do Rio dos Sinos. Os autores observaram uma dificuldade na mudança da abordagem tradicional para o PBL, porém, de modo geral, concluíram que os alunos ficaram bastante motivados e que desenvolveram habilidades para lidar com questões multidisciplinares e buscar soluções com base nos conhecimentos teóricos adquiridos, envolvendo a resolução de problemas, capacidade crítica, criatividade e o trabalho em equipe.

É possível destacar algumas pesquisas que apontam relatos da utilização da metodologia PBL no contexto dos cursos de Engenharia Civil. Macambira (2011) avaliou a adoção do PBL na disciplina "Gestão Empresarial" do curso de Engenharia Civil da Universidade Federal do Pará, no município de Belém. O pesquisador observou que os alunos reagiram positivamente à adoção da ferramenta de ensino, desenvolvendo habilidades como: capacidade de pesquisa, espírito empreendedor e busca por conhecimentos inovadores. O autor também concluiu que a metodologia é um instrumento interessante para o desenvolvimento profissional do docente. 
Milhomem et al. (2013) realizaram um estudo capaz de integrar os cursos de Engenharia Civil, Engenharia Elétrica e Engenharia Mecânica, interagindo com alunos do Ensino Médio de escolas estaduais do município de Tucuruí-PA. As atividades foram desenvolvidas por meio da apresentação de experimentos com baixo custo e utilizando materiais alternativos, envolvendo matemática, química e física. Os resultados apontaram para motivação dos alunos e aumento do senso de responsabilidade, contribuindo para que os mesmos pudessem expor os experimentos na $1^{a}$ Feira de Ciências das Escolas Públicas de Tucuruí-PA.

O trabalho de Lopes e Martins (2017) tinha por objetivo averiguar os resultados do uso da metodologia PBL no processo de ensino-aprendizagem relativo à disciplina Síntese e Integração dos Conhecimentos (SIC) que aborda a integração e o gerenciamento de projetos multidisciplinares de edificações na Engenharia Civil, em um curso da Região Sul. Os autores constaram que a metodologia PBL atuou na disciplina SIC de forma excelente, detectando maior aproximação dos alunos de situações práticas, uma melhor visualização entre teoria e prática, a cooperação e contribuição em grupos e a autoaprendizagem. No tocante ao trabalho em equipe os alunos desenvolveram competências de resolução de problema, tomada de decisão, respeito a opinião dos colegas, uma comunicação escrita e oral mais voltada a situações que serão encontradas em futuras reuniões profissionais.

Guimarães (2018) aplicou, de modo parcial, a metodologia PBL na disciplina de Cálculo Diferencial e Integral, para o curso de Engenharia Civil da Universidade Estácio de Sá, do Campus Cabo Frio. A autora menciona que a adoção da metodologia, por si só, não é capaz de solucionar todos os problemas do ensino-aprendizado, porém, mesmo com todas as dificuldades e desafios para sua implementação, os resultados foram positivos e capazes de aproximar teoria e prática.

Um relato de experiência da utilização da metodologia PBL detalha o desenvolvimento do conteúdo de Estações de Tratamento de Água na disciplina de Saneamento Ambiental em um curso de Engenharia Civil no Estado de Minas Gerais, realizado pelos autores Gonçalves e Aguilar (2019), o PBL foi utilizado como instrumento motivador e instigador. A discussão provida, levou a proposta pelos alunos de desenvolverem projetos para sanar os problemas detectados. Segundo os autores ao final da disciplina foi possível um aproveitamento satisfatório por parte da maioria dos alunos, alcançando níveis desejados tanto na avaliação escrita quanto no produto do projeto.

\subsection{A Engenharia Civil no município de Itaituba-PA}

A Ufopa foi criada pela Lei $n^{\circ} 12.085$, de 5 de novembro de 2009, sendo a primeira Instituição Federal de Ensino Superior com Sede no interior de um Estado da Amazônia brasileira. Sua Sede fica no município de Santarém e tem Campi nas cidades de Alenquer, Itaituba, Juruti, Monte Alegre, Óbidos e Oriximiná. Sua criação se deu a partir da política que instalou o Programa de Expansão das Universidades Federais do Programa de Apoio aos Planos de Reestruturação e Expansão das Universidades Federais (Reuni) e do acordo de cooperação técnica firmado entre o Ministério da Educação (MEC) e a Universidade Federal do Pará (UFPA) com o objetivo de ampliar o ensino superior na região amazônica (MULLER, 2019; UFOPA, 2020).

A estrutura acadêmica da Ufopa é focada na interdisciplinaridade, nas potencialidades regionais, e o Campus da Universidade na cidade de Itaituba apresenta potencial (hidrelétricas, ferrovias, portos de exportação, entre outros) para o desenvolvimento segmentado na 
sustentabilidade, na infraestrutura e na logística portuária, tendo em vista o desenvolvimento, com sustentabilidade, da região e os iminentes empreendimentos (UFOPA, 2020). No contexto de expansão do ensino superior no Brasil, o curso de Engenharia Civil, ofertado no Campus de Itaituba (CITB) da Universidade Federal do Oeste do Pará (Ufopa), surge como o primeiro curso de Engenharia Civil no município e o primeiro ofertado por uma instituição pública na região Oeste do Pará.

Além do curso de Engenharia Civil ofertado pelo CITB-Ufopa, o município de Itaituba também conta com a oferta do mesmo curso pela Faculdade de Itaituba (FAI), com a primeira turma ofertada no ano de 2020. Dessa forma, apesar de ser um curso tradicional no país, a Engenharia Civil ainda é um curso recente no município, com nenhuma turma formada ainda, evidenciando desafios quanto à formação de mão de obra local, principalmente no que diz respeito aos professores para atuar nesses cursos.

\section{METODOLOGIA}

Com o avanço da Covid-19 pelo país e, em específico, pelas cidades do interior, a suspensão das aulas presenciais foi uma das primeiras ações a serem tomadas pelas instituições, para tentar reduzir a contaminação causada pelo novo Corona vírus. Diante deste cenário, ações que viabilizassem o ensino à distância ou de maneira remota passaram a ser essenciais para continuidade das atividades acadêmicas.

\subsection{Utilização da metodologia PBL no CITB-Ufopa}

Nesse contexto, a Ufopa resolveu suspender o calendário acadêmico, fomentando a iniciativa de docentes para oferta de atividades extracurriculares, que pudessem ser aproveitadas pelos alunos dos seus cursos de graduação, sem prejuízo para os alunos que não tivessem acesso à infraestrutura adequada para realização de tais atividades, como cursos de extensão online, entre outras ações.

Dessa forma, os alunos de Engenharia Civil do CITB-Ufopa foram envolvidos em um projeto de extensão que trabalha com a produção e doação de totens para álcool em gel para postos de saúde, instituições que mantiveram o atendimento durante a pandemia e escolas para o retorno gradual às atividades, como forma de contribuir com o município de Itaituba no combate à Covid-19.

A produção dos totens se deu por meio de orientação remota, onde o docente apresentou aos alunos, por meio de um aplicativo de mensagens instantâneas, um modelo de totem produzido com canos de PVC, utilizados na construção civil, com acionamento pelo pé, sem a necessidade de tocar no dispenser de álcool em gel. Com essa informação, os alunos envolvidos no projeto passaram a pesquisar os materiais necessários e a forma de produção dos totens, realizando a fabricação dos mesmos em suas próprias casas e escolhendo as instituições para realizar a doação do equipamento, possibilitando que o aluno se tornasse ator principal da ação extensionista. A Figura 1 mostra o protótipo produzido. 
Figura 1 - Protótipo de totem para álcool em gel

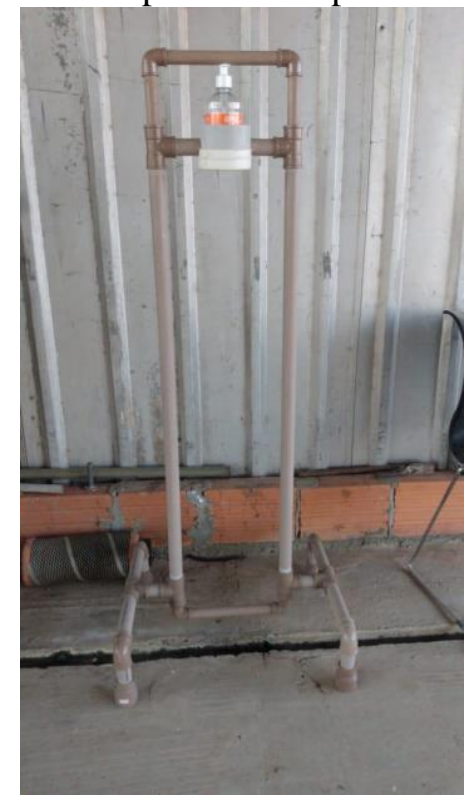

\subsection{Utilização da metodologia PBL na FAI}

Como forma de combater o avanço da Covid-19 no município de Itaituba, a FAI, inicialmente, passou a ofertar as aulas para os seus cursos de graduação à distância, com aulas virtuais. Após a publicação dos decretos municipal e estadual de flexibilização do isolamento social e reabertura gradativa das atividades econômicas, as aulas do curso de Engenharia Civil voltaram a ocorrer de maneira presencial e remota, com redução da quantidade de alunos na sala de aula.

Dentro da disciplina "Introdução à Engenharia", ofertada para os calouros, um dos componentes avaliativos se tratava da produção de totens de álcool em gel pelos alunos, divididos em grupos de 5 pessoas. Dessa forma, seria possível que os alunos pudessem desenvolver suas habilidades em projetar e executar os projetos desenvolvidos, de maneira autônoma e em grupos menores do que nas aulas convencionais. A adoção da metodologia PBL neste componente curricular já havia sido testada por Gezer et al. (2020), apresentando resultados satisfatórios.

O processo de desenvolvimento dos totens foi similar ao adotado pelos alunos do CITBUfopa, o docente apresentou um problema, onde os alunos precisariam encontrar uma solução. Dessa forma, os discentes da FAI pesquisaram qual seria a maneira mais prática e econômica de produzir um totem de álcool em gel, optando pelo modelo mostrado na Figura 1, buscando informações dos materiais e métodos necessários para a sua produção.

\section{RESULTADOS E DISCUSSÕES}

A metodologia PBL tem em sua essência possibilitar ao aluno se tornar ator principal no processo de ensino-aprendizagem, conferindo a ele habilidades para solucionar problemas cotidianos, mas também preparados para resolver problemas pontuais, como menciona Bringhenti (1993). Dessa forma, o projeto desenvolvido neste trabalho demonstrou a capacidade dos docentes e discentes em adotar tal metodologia para desenvolvimento da ação.

O projeto desenvolvido conferiu aos alunos o domínio pela produção do conhecimento e sua aplicação efetiva na sociedade, por meio da entrega de equipamentos que são eficientes no 
combate à disseminação do novo Corona vírus. A Figura 2 mostra alguns dos totens produzidos pelos alunos dos cursos de Engenharia Civil do CITB-Ufopa e da FAI.

Figura 2 - Totens produzidos pelos alunos de Engenharia Civil de Itaituba
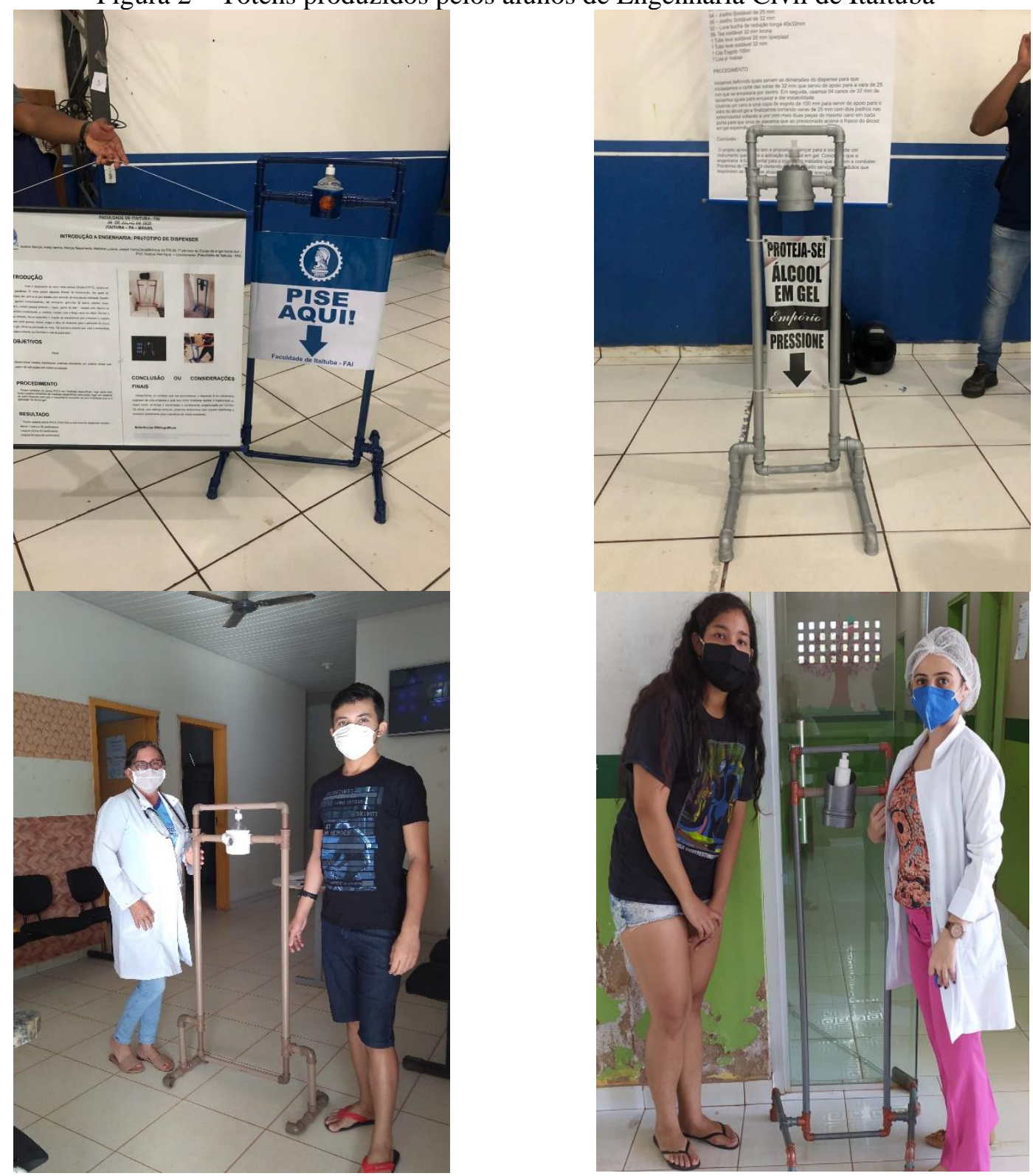

Em relação ao processo de ensino-aprendizagem, no processo avaliativo da FAI observouse um bom desempenho dos alunos, verificando-se um bom nível de conhecimento por parte dos mesmos quanto ao contexto de pandemia, bem como quanto ao processo de produção dos equipamentos. Também foi possível observar o desenvolvimento de habilidades para trabalho em grupo, elaboração e execução de projeto, interação com a sociedade e resolução de problemas.

Quanto aos alunos da Ufopa, por se tratar de uma atividade de extensão, o processo não foi avaliativo, mas foi possível ver um certo domínio e aprendizado dos discentes por meio de seus relatos. Além de desenvolverem competências para solucionar problemas e melhorar a autonomia no processo de aprendizagem, os mesmos também foram capazes de liderar equipes, 
muitas vezes formadas pelos familiares de suas residências, que também contribuíram para a produção dos totens.

Vale ressaltar que, além das habilidades desenvolvidas e reconhecidas pela metodologia PBL, os discentes envolvidos nessa ação tiveram a oportunidade de interagir com diversos setores da sociedade, desde as instituições atendidas pelas doações dos totens, até as empresas de engenharia e materiais de construção do município de Itaituba, que contribuíram com a doação de materiais para produção dos totens. Assim, o projeto realizado possibilitou envolvimento social com a comunidade, aliado ao espírito inovador e empreendedor.

Quanto ao contexto da pandemia, a metodologia PBL se mostrou bastante eficiente para continuidade ou retomada das atividades acadêmicas, visto que possibilita maior autonomia do aluno quanto ao ensino remoto, dentro de suas próprias realidades de tempo, infraestrutura e condições sociais, assim como contribuiu para evitar a aglomeração por conta do elevado número de alunos em sala de aula, como no modelo tradicional de ensino.

\section{CONSIDERAÇÕES FINAIS}

Este trabalho apresentou uma abordagem sobre a metodologia PBL, quanto aos seus conceitos, desafios e relatos de pesquisadores sobre a sua aplicação nos cursos de Engenharia Civil. Também foi abordado sobre o contexto da própria Engenharia Civil no município de Itaituba-PA, para possibilitar uma melhor compreensão dos desafios encontrados para adoção dessa ferramenta e viabilidade do seu uso nos cursos de Engenharia Civil desse município.

Para avaliar a viabilidade do PBL nos cursos de Engenharia Civil ofertados em ItaitubaPA, foram realizadas ações dentro de um projeto com alunos da Ufopa e FAI, onde se verificou que as ações executadas atenderam ao que a própria metodologia prima: desenvolvimento do senso crítico, capacidade de solucionar problemas, habilidades para trabalho autônomo e em grupo, inovação e empreendedorismo.

Quanto ao contexto da pandemia, avaliou-se que a metodologia PBL é bastante eficiente para garantir um ensino de qualidade aos alunos, visto que, como forma de evitar a propagação do vírus, ações como atividades remotas e redução do número de alunos por sala de aula passam a ser parte do novo cenário pós-pandemia.

Vale ressaltar que uma das maiores dificuldades para implantação do PBL é quanto a competência dos docentes e, de acordo com Gonçalves (2020), o exercício da docência pelo professor bacharel torna necessária a busca por formações complementares, assim, é de extrema importância o envolvimento das instituições de ensino para implantação da ferramenta em seus cursos, por meio de ações e capacitação dos seus docentes, bem como alguns resultados podem levar mais tempo para serem alcançados, como lembra Guimarães (2018).

\section{Agradecimentos}

Os autores agradecem à Universidade Federal do Oeste do Pará (UFOPA), ao Campus Universitário de Itaituba (CITB) e à Faculdade de Itaituba pelo apoio e fomento à realização deste trabalho. 


\section{REFERÊNCIAS}

BRINGHENTI, I. O ensino na escola politécnica da USP: Fundamentos para o ensino de engenharia. São Paulo: ESPUSP, 1993.

GEZER. et al. Aplicação da metodologia PBL (problem based learning) em uma disciplina inicial dos cursos de Engenharia Civil. Série Educar - Ensino Superior, V. 16, Cap. 6, p. 3843, 2020.

GONÇALVES, R.C.C. As práticas pedagógicas do professor bacharel no Ensino Superior. Dissertação (Mestrado em Ensino) - Universidade do Vale do Taquari: RS, 2020.

GONÇALVES D. K. C. e AGUILAR M. T. P. Metodologias ativas aplicadas na disciplina de saneamento ambiental no curso engenharia civil. Brazilian Journal of Development, V. 5, n. 10, p. 19315-19326. Curitiba, 2019.

GUIMARÃES, G. Aprendendo cálculo diferencial e integral em engenharia civil: uma proposta interdisciplinar entre teoria e prática. Revista de Ensino de Engenharia, v. 37, n. 1, p. 66-75, 2018.

LOPES, A. P.; MARTINS, D. B. PBL como estratégia de ensino-aprendizagem em uma disciplina de integração e gerenciamento de projetos multidisciplinares de edificações na engenharia civil. Gestão \& Tecnologia de Projetos, São Carlos. v.12 n.1 p 53-67, 2017.

MACAMBIRA, P. M. F. A aprendizagem baseada em problemas (ABP): uma aplicação na disciplina "Gestão Empresarial" do curso de engenharia civil. Dissertação Mestrado, Instituto de Tecnologia, Universidade Federal do Pará, Belém, 2011.

MASSON, T.J., et al. Metodologia de ensino: aprendizagem baseada em problemas (PBL). Anais do XL Congresso Brasileiro de Educação em Engenharia, Belém, 2012.

MILHOMEM, P.M., et al. Aplicando a metodologia PBL na região Amazônica para incentivo ao Estudo de Engenharia. Anais do XLI Congresso Brasileiro de Educação em Engenharia, Gramado, 2013.

MULLER, Fabiano Hector Lira. A implantação do PARFOR/UFOPA no município de Itaituba/PA: indicadores e resultados. Dissertação (Mestrado em Ensino) - Universidade do Vale do Taquari: RS, 2019.

NEVES, R.M.; FORMOSO, C.T. Aprendizagem baseada em problemas: estudo exploratório com alunos de graduação em cursos de engenharia civil. Anais do XXXV Congresso Brasileiro de Educação em Engenharia, Curitiba, 2007.

RIBEIRO, L. R. C. A aprendizagem baseada em problemas (PBL): uma implementação da educação em engenharia na voz dos atores. Tese de Doutorado, Programa de PósGraduação em Educação, Centro de Educação e Ciências Humanas, Universidade Federal de São Carlos. São Carlos, 2005. 
RIBEIRO, L. R. C. Aprendizagem baseada em problemas (PBL) na educação em engenharia. Revista de Ensino de Engenharia, v. 27, n. 2, p. 23-32, 2008.

\title{
UFOPA - UNIVERSIDADE FEDERAL DO OESTE DO PARÁ. Plano de Desenvolvimento Institucional da Ufopa 2019-2023. 2020. Disponível em: <http://www.ufopa.edu.br/media/file/site/proplan/documentos/2020/766ea1d5a36f6bde3acdc 4b46199218e.pdf>. Acesso em: 09 jul. 2020.
}

VIEIRA, K. A utilização do PBL nos cursos de engenharia no Brasil: uma análise bibliométrica. Trabalho de Conclusão de Curso, Curso de Pós-graduação Lato Sensu em Ciências e Tecnologia, Universidade Federal de Santa Catarina, Joinville, 2017.

\section{PBL METHODOLOGY AS AN ALTERNATIVE FOR TEACHING FEASIBILITY IN COVID-19 PANDEMIC CONTEXT}

\begin{abstract}
This work presents a bibliographic review on the use of the PBL methodology in Civil Engineering courses, as well as a context of the Civil Engineering course itself in the municipality of Itaituba-PA, to enable a better understanding of the challenges involved in adopting this tool in this reality. The main objective of this work focuses on the adoption of PBL as an alternative for continuing education in the context of the pandemic by Covid-19, as well as the possibility and challenges for using this method within the Civil Engineering courses offered in the municipality of Itaituba. To obtain the results, actions were carried out involving two HEIs, through the production of totems for alcohol gel, in order to allow the development of works by students from both institutions, generating a product for society. It was concluded that the PBL methodology is an efficient tool to enable quality teaching in the context of pandemic and post-pandemic and that, despite the challenges, it is possible to use it in the studied courses.
\end{abstract}

Keywords: PBL Methodology. Civil Engineering. Pandemic. Itaituba. 